\title{
Active Performance Measurement for IP over All-Optical Networks
}

\author{
Marat Zhanikeev*, Sugang $\mathrm{Xu}{ }^{\dagger}$, and Yoshiaki Tanaka*‡, \\ * Global Information and Telecommunication Institute, Waseda University \\ 1-3-10 Nishi-Waseda, Shinjuku-ku, Tokyo, 169-0051 Japan \\ Email: maratishe@asagi.waseda.jp \\ $\dagger$ National Institute of Information and Communications Technology \\ 4-2-1 Nukuikitamachi, Koganei-shi, Tokyo, 184-8795 Japan \\ * Advanced Research Institute for Science and Engineering, Waseda University \\ 17 Kikuicho, Shinjuku-ku, Tokyo, 162-0044 Japan
}

\begin{abstract}
It is predicted that networks in the near future will consist mostly of optical components in order to satisfy growing demands for bandwidth. Although the model of a global all-optical network is not clear in details yet, many researchers are certain that a shift of communication paradigm is to take place. In the world of electric networks, the concept of active measurement is very well established and is proved to be quite successful for realtime monitoring of network performance. Alloptical networks will require a different approach to active performance measurement. The details of a measurement model applicable to optical networks and its differences from traditional measurement are the main subjects of this paper.
\end{abstract}

\section{INTRODUCTION}

Optical networks are currently considered the best technology candidate for the next-generation Internet [1] [2] [3]. They are expected to fulfill the requirements for bandwidth from emerging network-oriented applications and services. Until recently, however, circuit-switched optical components, being intrinsically fairly cheap, were expected to replace expensive ATM switches. As both optical OXC (optical cross-connects) and ATM meshes have similar functionality, such a replacement allowed easy implementation.

Presently, however, research in optical networks is looking for replacements for mostly all network devices used in electric networks [4]. Even the vested lack of optical memory in routers is dealt with by introducing Optical Delay Lines (ODL), which to some extent can serve as limited size buffers for optical packets.

Along with the interest in replacing devices commonly used in electric networks with their optical substitutes, the concept of all-optical network was introduced as the environment in which all buffering, switching, and routing is performed in optical domain. If optical networks will be fully in charge of global networking, they will also have to inherit obligations of electric networks. One major obligation is the assumption that in electric networks realtime performance, i.e. throughput, outages, available bandwidth, delay jitter, etc., can be inferred through end-to-end active measurements. Endto-end performance measurement is the key technology in global grid and other distributed network-centric applications and services, which cannot establish adhoc topologies among multiple nodes without realtime snapshots of performance in the underlying network. Detailed description of advanced in end-to-end active probing basics can be found in [5]

This paper studies new features in measurement results obtained in all-optical networks using conventional multi-packet probes. The optical network used in simulation tests contains all modern advances in optical burst switching (OBS) and optical packet switching (OPS) [6]. A model of a global optical network is created with active probing performed through the optical infrastructure. Finally, after analysis of measurement results, features of a new measurement model suitable for all-optical networks are discussed.

\section{Performance Measurement in Optical NETWORKS}

\section{A. Traditional Performance Measurement}

Traditionally, all active measurement methods are based on the dispersion of interarrival time between neighboring packets in a multi-packet probe [5]. The dispersion can be studied in the most trivial case of a packet 


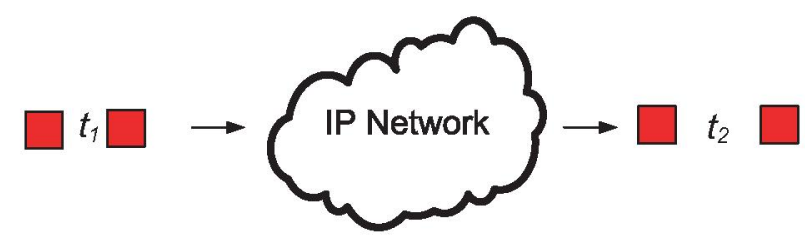

Fig. 1. Traditional measurement model based on packet interarrival time.

pair, which is the building block of any active measurement technique. The model of active measurement based on time dispersion in packet pairs is displayed in Fig.1.

As measurement theory has it, when $t_{1}$ in Fig. 1 is small enough to allow packets to queue back to back at the narrowest link in the network, the $t_{2}$ is enough to infer the bottleneck bandwidth, i.e. the narrowest link in the path. The bottleneck bandwidth is calculated as simple $S / t_{2}$, where $S$ is the size of probing packets. The packet-pair property is valid only if both packets in the probe are of the same size and if they queued back to back at the bottleneck.

There are multiple variations of packet-pair, some of which along with the relation of properties in probing results to the design of the probe can be found in [5]. In present practice, however, the most attention is given to available bandwidth, where measurement methods normally use long packet trains and do not specifically use packet-pair properties, although still using it to define ranges for measurement parameters.

One of the major problems today for any active measurement method is high transmission rates in links. The higher is the transmission rate, the higher is the error in measurement results. In fact, links above $1 \mathrm{Gbps}$ are rarely considered in measurement literature. In this respect, optical networks pose yet a greater challenge to probing, as the capacity of optical links is normally well above 1Gbps. The capacity of several terabit per second was also occasionally reported in literature on optical networks.

However, high transmission rates is not the only challenge performance measurement is to encounter in all-optical networks. Another major challenge is traffic aggregation that is proposed as replacement to commonly used today packet-by-packet routing.

\section{B. Aggregation in Optical Networks}

Fig. 2 describes two major switching techniques proposed as replacement for traditional packet-by-packet routing in all-optical networks [4] [?]. OPS is a direct replacement of traditional electric packet switching, as

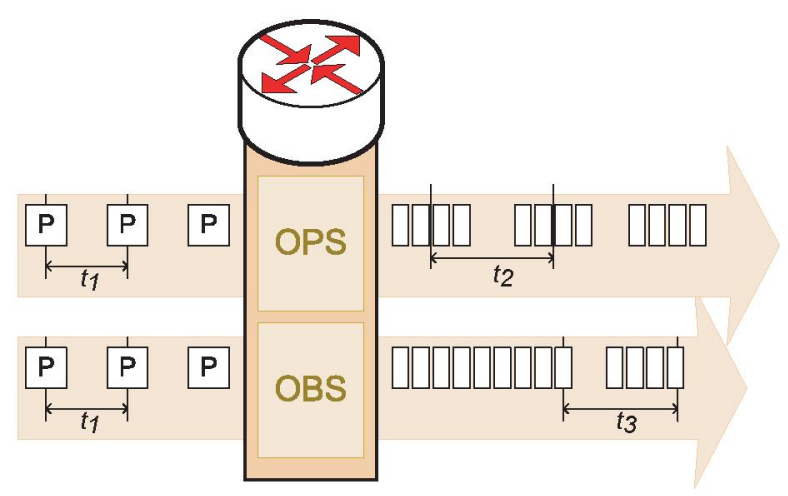

Fig. 2. Model of influence to probing by aggregation in optical networks.

it routes packets one by one based on the header that is also optically processed. Buffers in OPS are emulated by ODL modules of limited size. Compared with un-slotted OPS, it is believed that slotted OPS will have better performance in terms of packet loss rate. Although it will introduce extra cost for packet synchronization [6]. Here we assume the slotted type of OPS.

At the entrance into the all-optical domain, optical packets are split into smaller optical frames each of which is transmitted within a fixed-length transmission slot, that is a common manner of communication in optical networks.

Regardless of the fact that a large IP packet is fragmented and transmitted over a number of consecutive slots, due to very high bandwidth of optical links, we can assume that optical frames will be separated by the interval $t_{2}$, which will be equal to $t_{1}$ with a negligible surplus introduced by possible buffering within OPS.

OBS module performs differently from OPS and is based on traffic aggregation [4]. Aggregation is performed in traffic flows, i.e. groups of packet with the same source and destination address pairs, and can be implemented either in regular transmission, i.e. using timeout for aggregated flows, or in thresholds applied to size of an individual flow. After the flows are ready for transmissions, a traffic burst is scheduled in accordance with one of many existing proposals for burst scheduling, i.e. Just In Time (JIT), Just Enough Time (JET), Tell And Go (TAG), and others.

We expect different properties in measurement data for OPS and OBS. In case of OPS, the measurement data will not have many properties in form of irregularities in measurement data. In case of OBS, bursty nature of traffic aggregation may offer some room for creating new measurement properties. The properties and a new model 


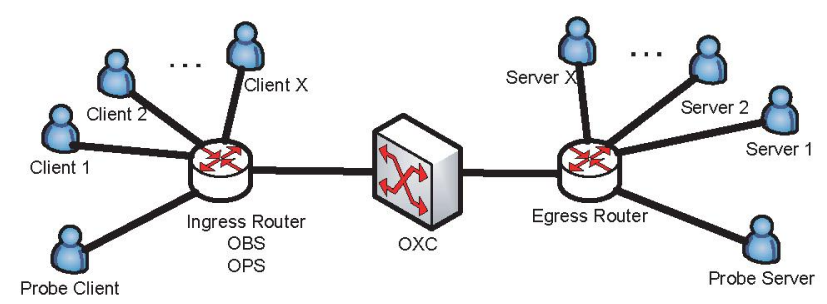

Fig. 3. Network topology used for simulation.

of measurement in optical networks are discussed in the next section.

\section{Simulation REsults}

\section{A. Network Topology}

Fig. 3 displays a simplified network topology used for simulation. The simulation is performed using OPNET Modeler. As commonly used in modeling of global all-optical networks [7], the network topology contains entrance node that aggregates and adapts traffic to the internal optical logic of communication. The internal switching is performed by optical cross-connect (OXC) node. Finally, before exiting back to the electric domain, optical frames are again aggregated using egress router and finally transmitted into the IP domain in form of IP packets.

We use multiple client-server pairs that generate Pareto ON/OFF traffic with the following stochastic setup. Length of OFF period and traffic size of ON period are made random with shape parameter $c=1.2$ and location $a=5000$ for traffic size in ON period measured in bytes and location $a=0.1$ for OFF period measured in seconds. Traffic size of ON period is split in equally sized packets taken from uniform distribution in the range from 60 to 1500 bytes with a different random number taken for each ON period. Packets are transmitted with a static interval of 5 microseconds.

For measurement we use probes of 100 packets each 1000 bytes long and equally spaced every 5 microseconds. Individual probes are transmitted every second and analyzed at the receiving end. All optical links in the network have the capacity of $1 \mathrm{~Gb}$, and all IP links are $100 \mathrm{Mbps}$. However, as each client/server and in particular the probing pair are using separate links to connect to the optical environment, the bandwidth in IP is grossly underutilized most of the time.

\section{B. Probing results in OPS and OBS environments}

As the main purpose of the present study is finding measurement properties that are new and specific to

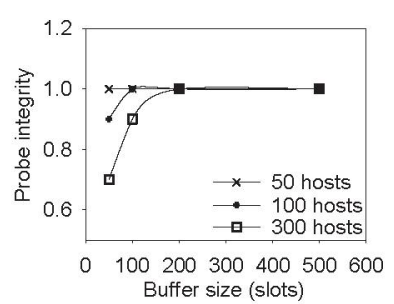

(a) Probe integrity

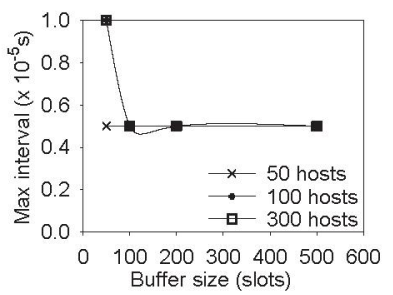

(c) Maximum interval in probe

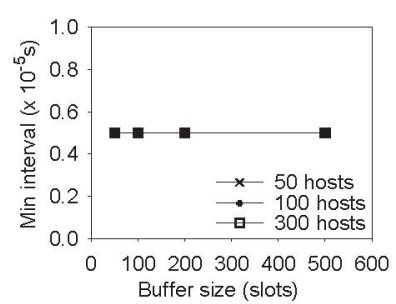

(b) Min interval in probe

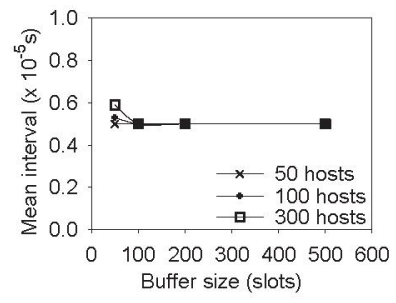

(d) Mean interval in probe
Fig. 4. Measurement data obtained in OPS-routed optical environment.

optical environment, all data pertain to statistics collected from probes arriving at the other end of measurement path. We use four main metrics : probe integrity, which is to indicate packet loss within the probe and grows lower as more probing packets are lost, minimum, maximum and mean interval found between neighboring packets. Min and max values are single readings in each probe, while mean is a mean of all intervals between packets, i.e. is the mean of 99 samples for each probe, as we use 100-packet probes. To exclude random error, each sample is the average of 10 separate runs of simulation.

Results in Fig. 4 are obtained using OPS optical environment. As was already mentioned before, OPS is the closest optical technology to traditional packet switching. However, according to the results, OPS-switched networks are very robust even when heavily utilized. In fact, in Fig.4(a), the integrity of the probe never fall below 0.7 even with 300 noise-generators to interfere with, i.e. even such heavy utilized conditions result in the loss of only $30 \%$ of packets in the probe.

However, as can be seen from Fig.4(c), the packet loss normally is very local, i.e. only 1-2 packets are missing in a row, which, thus, does not change the max interval too much. This conclusion is supported by Fig.4(d), where there is very minor difference in mean interval values even with some packet loss.

This property of OPS can be explained by considerable difference in link capacities before and after OPS. This results in very short-time bursts of traffic, in the middle of which some packets may be dropped. 


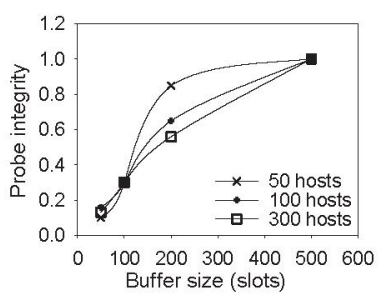

(a) Probe integrity

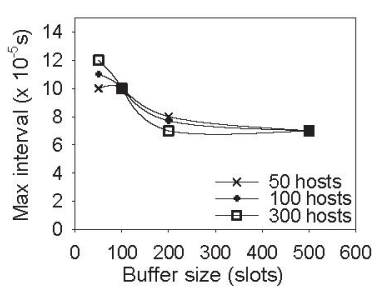

(c) Maximum interval in probe

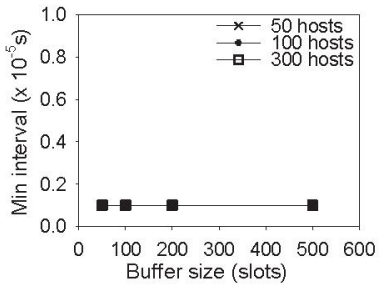

(b) Min interval in probe

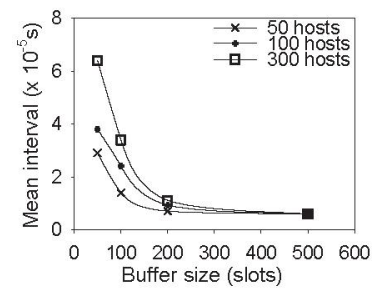

(d) Mean interval in probe
Fig. 5. Measurement data obtained in OBS-routed optical environment with bursts created using $0.1 \mathrm{~ms}$ timeout.

As measurement packets are transmitted at steady 5microsecond intervals, they cannot cause congestion at the buffer of OPS, but can be found in the middle of background traffic burst with a certain low probability. Since the probability of this event is low, the measurement results do not offer any major irregularities even under heavy utilization.

Therefore, the major feature that can be attributed to OPS is fast recovery, which make is unlikely that two probing packets will be included in the same queue. It should be mentioned that traditionally measurement methods consider the whole probe to be included in a single queue with limited interference with background traffic.

Fig.5 contains results obtained in OBS optical environment using timeout-based bursts. In optical plain we use Just Enough Time (JET) logic of burst scheduling. Timeout of $0.1 \mathrm{~ms}$ is used specifically to allow probing packets to be split into 5 consequent bursts. The major point of interest therefore is to visualize interference of separate bursts within a single probe with background traffic, that is also aggregated in bursts of unknown length (Pareto distribution of ON sizes).

As can be seen from Fig.5, OBS with timeout resulted in non-linear properties in probing results. In fact, in terms of probe integrity, the non-linearity is alleviated with growth of the number of noisy hosts. The lowest point of probe integrity is almost the same for all curves and stands for $10 \%$ of successfully received packets, i.e.

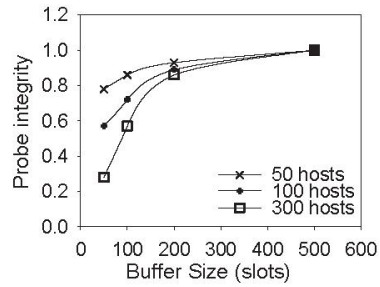

(a) Probe integrity

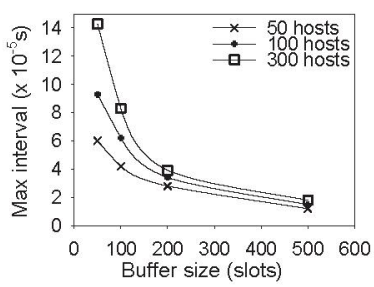

(c) Maximum interval in probe

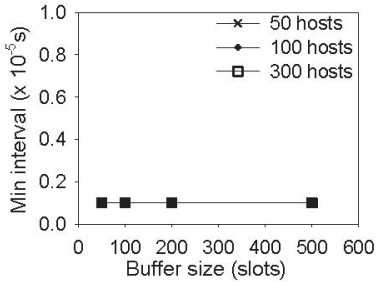

(b) Min interval in probe

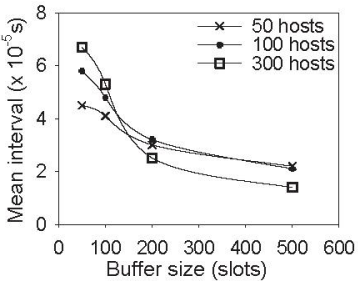

(d) Mean interval in probe
Fig. 6. Measurement data obtained in OBS-routed optical environment with bursts created using $20 \mathrm{kB}$ threshold.

heavy drop. This is a natural outcome if to consider that with timeout-based bursts the size of each burst is not defined and depends upon random setup of each background traffic host. This is the reason of heavy loss of probing packets.

The min interval remains steady for all conditions and is lower than was in the case of OPS, since in bursts, probing packets are compressed back-to-back in a single burst. Also because of burstiness, the maximum interval is 2 orders of magnitude higher than minimum interval, which indicates of high variance of intervals among packets in the probe. In fact, mean intervals are also fairly high and exhibit non-linear response to different simulation conditions.

Yet a different pattern of irregularity in probing data is exhibited in Fig.6, which contains data from simulation performed in OBS environment with bursts created using the size threshold of $20 \mathrm{kB}$, i.e. again in such a way that a single probe is split into 5 separate bursts. Although probing-wise, the conditions for creating bursts are identical, interference of probing bursts with background traffic is different.

In fact, OBS with size threshold behaves similar to OPS. Probe integrity in this case grows fast with larger buffers, quickly converging to zero loss. Bursts interfere with background traffic in a similar fashion to OBS with timeout, which is the reason for high variance in packet interval readings. The maximum intervals are in the same range as in OBS with timeout, but exhibit more non- 
linear behavior, quickly dropping with increase of buffer size.

Curves of mean interval in Fig.5(d) and Fig.6(d) are also different, which indicates of the fact that gaps in probe are situated differently for OBS with timeout and OBS with size threshold. The most non-linear part at low values of buffer size is more important as buffers in optical routing equipment are considerably smaller than traditionally expected in electric equipment. Results in OBS with size threshold are exhibit slow convergence in this area which proves that size-threshold OBS causes more interference and therefore, higher irregularity in probing data.

Generally, results obtained from OBS appear to cause more irregularities in probing data than OPS. However, it should be noted that most differences are in the range of a few dozen microseconds, which is very close to the limits of precision that operating systems can offer in timestamps. This is to say that although techniques used in optical routing cause different patterns of interference, the differences in packet timestamps when returned back to electric domain, are too minor to perform any serious statistical study.

\section{CONClusions}

This paper discussed properties of active performance measurement when applied specifically to all-optical networks. As the optical part in global networks is expected to grow, a model for performance measurement based on active probing, i.e. without access to the actual networking infrastructure, will be needed by networkcentric applications, such as global grid networks, distributed data processing, and others.

Obviously, the model for active measurement in alloptical networks should be very different from traditionally used in electric IP networks. This paper performed a simulation study in order to identify original properties that exist only in optical networks and can be exploited to infer performance of optical networks preferably in realtime.

As was displayed by simulation results, from two major routing technologies proposed for next-generation optical networks, OPS does not seem to offer any tangible irregularities in probing data to exploit even with help of mathematical statistics. OBS technology, on the other hand, results in fairly irregular timestamps of probe packets at the receiving end. These irregularities, however, are places in the range of dozens of microseconds and will be even less with increase of capacity in optical domain.
Taking into considerations the data obtained in the present paper, it is obvious, that none of the traditional active measurement assumptions work in optical domain, least of all the main packet-pair assumption that uses the space between two neighboring packets. In the optical domain, measurement model will have to consider a large number of packets as a single chunk disregarding the space between the individual packets. To facilitate this, probes will have to be considerably longer than commonly used in traditional measurement. A probe of a few thousand packets should not be unusual in optical domain. Alternatively, packets may be transmitted into optical network with a large interdeparture time. This will result in different treatment of each packet in bursty parts of optical networks.

The above can be finalized with the conclusion that the most robust measurement model in optical network is a hybrid of probes consisting of many back-toback packets and individual single-packet probes. Singlepacket probes will allow to infer general conditions of the network, while multiple-packet probes will exploit bursty properties of optical network. Packet loss should not offer much room for performance inference, as with proper implementation of IP over WDM using any of many currently proposed technologies, packet loss in optical domain should be limited and will not be more than can be found presently in electric IP networks.

In the future study, we plan to finalize a model of active performance measurement in a set of probing methods and methods of performance inference based on mathematical statistics.

\section{REFERENCES}

[1] K. M. Sivalingam and S. Subramaniam, Optical WDM Networks : principles and practice. Kluwer Academic Publisher, 2002.

[2] G. N. Rouskas and H. G. Perros, "A tutorial on optical networks," in Advanced Lectures on Networking, London, UK, May 2002, pp. 155-194.

[3] M. J.O'Mahony, D. Simeonidou, D. K.Hunter, and A. Tzanakaki, "The application of optical packet switching in future communication networks," IEEE Communications Magazine, vol. 39(3), pp. 128-135, March 2001.

[4] J. Serrat and A. Galis, Deploying and managing IP over WDM networks. Artech House, 2003.

[5] X. Liu, K. Ravindran, and D. Loguinov, "What signals do packetpair dispersions carry?" in INFOCOM, vol. 1. IEEE, March 2005, pp. 281-292.

[6] S. Dixit, IP over WDM: building the next-generation optical Internet. John Wiley\&Sons, 2003.

[7] M. Duser and P. Bayvel, "Modelling of optical burst switched packet networks," in Workshop on Modelling and Design of Optical Networks and Systems (ECOC), Munich, Germany, September 2000. 